\title{
Characteristics and outcomes of patients screened by rapid response team who transferred to the intensive care unit
}

\author{
Song-I. Lee, Jeong Suk Koh, Yoon Joo Kim, Da Hyun Kang and Jeong Eun Lee* (1)
}

\begin{abstract}
Background: The utilization of a rapid response team (RRT) has influenced the clinical outcomes of patients in the general ward. However, the characteristics of RRT-screened patients who are transferred to the intensive care unit (ICU) are unknown. Therefore, the present study aimed to evaluate these factors.
\end{abstract}

Methods: We conducted a retrospective study using patient data from a tertiary medical center in Republic of Korea between January 2016 and December 2017. Multivariate logistic regression analyses were performed to assess the factors associated with the risk of in-hospital mortality.

Results: A total of 1,096 patients were included: 389 patients were transferred to the ICU, and 707 patients stayed in the ward. Patients in the ICU group were more likely to be admitted for medical reasons, hepatobiliary disease, and high heart rate. More interventions were performed, hospital stays were longer, and the 28-day and in-hospital mortality rates were higher in the ICU group than in the ward group. Multivariate logistic regression analyses showed that risk factors affecting ICU admission were higher Sequential Organ Failure Assessment (SOFA) score, National Early Warning Score (NEWS), platelet count, and lactate level. ICU transfer was not associated with in-hospital mortality.

Conclusions: Among RRT-screened patients, those with higher SOFA score, NEWS, and lactate level were more likely to be transferred to the ICU. Therefore, these patients should be closely monitored and considered for ICU transfer.

Keywords: General ward, Intensive care unit, Prognosis, Rapid response team

\section{Background}

A rapid response for general ward patients suffering from acute deterioration may be impossible because of missing symptoms and unrecorded vital signs. However, regular monitoring and introduction of automatic alarm systems aid the emergency medical team, which operates $24 \mathrm{~h}$ or part-time a day, to implement treatment and reduce mortality [1-3]. General ward patients (up to $10 \%$ of cases) experience unexpected events [4], and $7.3 \%$ of them experience fatal events [5]. Rapid response team (RRT)

\footnotetext{
*Correspondence: jelee0210@cnu.ac.kr

Department of Pulmonary and Critical Care Medicine, Chungnam National University School of Medicine, 33 Munhwa-ro, Jung-gu, 301-721 Daejeon, Republic of Korea
}

activation is usually triggered by several factors, such as monitoring of vital signs, pre-rounding, and direct calls from attending physicians, nurses, and family members $[3,5]$.

However, some studies have shown that having an RRT in the hospital is associated with higher intensive care unit (ICU) admissions and fewer severe patient transfer from the ward [6]. In addition, RRT intervention does not improve the disease severity and outcomes of patients transferred from the ward [6,7]. These studies have usually compared the characteristics before and after the RRT intervention. Patients transferred to the ICU after RRT screening, and prognostic factors for RRTscreened patients are not well known. Thus, the present 
study investigated the characteristics and outcomes of patients who were transferred to the ICU among patients screened by RRT.

\section{Methods}

\section{Study design and patient selection}

This was a retrospective observational study of patients admitted to Chungnam National University Hospital, a 1200-bed tertiary academic hospital in South Korea, between January 2016 and December 2017. Patients with a Do Not Resuscitate (DNR) record were excluded because they affected the availability of interventions such as invasive mechanical ventilation and admission to the ICU [8-10].

\section{Rapid response team}

We started operating an RRT at our hospital for adult patients from 7 AM to 11 PM daily on weekdays in 2014. The RRT consists of 3 ICU staff (ICU) and three dedicated nurses with experience in critical care. At least one intensivist and one dedicated nurse were on duty every day. The RRT was equipped for monitoring and resuscitation of airway, breathing, and circulatory emergency (patient monitor, emergency drugs, videolaryngoscope, point-of-care-testing arterial blood gas analysis, portable ultrasonography, and portable ventilator).

We screened adult patients over 18 years of age, excluding pediatric patients. Elderly and obstetric patients were included in the screening. Screening criteria from electronic medical records (EMRs) were as follows: systolic blood pressure $\leq 80 \mathrm{mmHg}$, respiratory distress (rate $\geq 30$ breaths/min), saturation of percutaneous oxygen $\left(\mathrm{SpO}_{2} \leq 85 \%\right)$, sudden mental change, or unexplained agitation. Admitted patients' vital signs were checked regularly by nurses in the ward on every duty (every $8 \mathrm{~h}$ ). If the patient in the ward was deteriorating, then vital signs were checked and recorded more frequently at 10-min to 1-h intervals. In addition, the EMR-based National Early Warning Score (NEWS) system was established in November 2013 and updated in December 2017, and it is used for adult patients admitted to the general ward [11]. The NEWS system is based on six physiological parameters: respiration rate, oxygen saturation, systolic blood pressure, pulse rate, level of consciousness or new confusion, and temperature. When a nurse in duty puts the above six parameters on the vital sign sheet of EMR, the score is automatically calculated. When the nurses of the ward recorded the vital signs in real time, if there was a vital sign abnormality or NEWS of more than 5 points [12], then the patient list and abnormal findings could be checked by RRT nurses.

The RRT screening was performed when 1) the measurements of a patient exceeded the pre-defined thresholds in the EMR-based automatic screening system, 2) doctors or nurses called the RRT for aid, or 3) code blue was announced for cardiopulmonary arrest.

ICU admission for patients screened at the RRT was decided by the intensivist in charge of the RRT at that time. Even when the RRT did not screen, the same intensivist decided whether to admit the ICU.

\section{Data collection}

All study data were retrieved from the EMR (C\&U Care, Daejeon, Republic of Korea). A total of 1,218 patients were screened by RRT between February 1, 2016, and December 31, 2017. The study enrolled 1096 patients, excluding 122 patients with DNR. Demographic, clinical, and radiological information, as well as laboratory and imaging data, was collected. Interventions performed at RRT included those performed at the ward and those performed within $24 \mathrm{~h}$ of admission to the ICU after deciding to be performed at the RRT.

Sequential Organ Failure Assessment (SOFA) scores based on Vincent et al. were used to predict mortality during the first $24 \mathrm{~h}$ of ICU admission [13]. The worst value was chosen for each organ system every $24 \mathrm{~h}$ to calculate the score [13]. The NEWS system was used for making acute illness assessments and determining responses [14]. The reason for RRT screening was defined by referring to the test findings of the patients at the time of screening for RRT and the patient's vital signs and patient's history.

Ethics approval.

This study was approved by the Institutional Review Board (IRB) (IRB No: CNUH 2019-06-030), and the requirement for informed consent was waived because of the retrospective nature of the study.

\section{Statistical analysis}

All values are expressed as mean \pm standard deviation and median and interquartile ranges for continuous variables and percentages for categorical variables. Student's t-test or the Mann-Whitney U test was used for continuous data, and Pearson's chi-square test or Fisher's exact test was used for categorical data analysis. Predictors of disease severity were identified through univariate logistic regression analysis. Multivariate logistic regression analyses with a backward elimination procedure, including all predictors with a $\mathrm{p}$-value of $\leq 0.05$ in the univariate analysis, were performed to obtain the adjusted odds ratio (OR) along with $95 \%$ confidence interval $(\mathrm{CI})$ and to determine the variables independently associated with disease severity. All $p$-values were two-tailed, and $\mathrm{p}$-values of $<0.05$ were considered statistically significant. All statistical analyses were performed using SPSS software (version 22.0; IBM Corporation, Somers, NY, USA). 


\section{Results}

\section{Patients' baseline characteristics}

Of the total 1096 enrolled patients, 707 (64.5\%) stayed in the ward and 389 (35.5\%) were transferred to the ICU (Fig. 1). The characteristics of the 1,096 patients are presented in Table 1. More patients were admitted for medical reasons $(75.8 \%$ vs. $70.3 \%, p=0.050)$ among those admitted to the ICU (ICU group). There were fewer cases of chronic lung disease ( $9.3 \%$ vs. $15.4 \%, p=0.004)$, and chronic hepatobiliary disease $(12.1 \%$ vs. $7.8 \%, p=0.019)$ was the more common underlying disease in the ICU group. There were no significant differences in other underlying diseases.

Vital signs recorded when the RRT first screened the patient showed that the heart rate was faster in the ICU group (106 [90-124] vs. 102 [88-116], beats/min, $p=0.002)$. Laboratory findings showed that the platelet count was lower $\left(170\right.$ [101-250)]vs. $192[123-266], \times 10^{3} /$ $\mu \mathrm{L}, p=0.018)$, and T-bilirubin [0.80 (0.50-1.40) vs. 0.70 (0.45-1.19), $\mathrm{mg} / \mathrm{dL}, p=0.018]$, creatinine $(0.97[0.71-$ $1.90]$ vs. $0.78[0.59-1.24], \mathrm{mg} / \mathrm{dL}, p=0.002)$, and C-reactive protein (CRP) levels (9.0 [2.8-17.6] vs. 8.5 [2.9-15.7] $\mathrm{mg} / \mathrm{dL}, p<0.001)$ were higher in the ICU group. The SOFA score (5 [3-7] vs. 3 [2-5], $p<0.001)$ and NEWS $(8[6-10]$ vs. $7[6-9], p<0.001)$ were higher in the ICU group. Other vital signs and laboratory findings showed no significant differences between groups (Table 1).

\section{Intervention and Outcomes}

Table 2 showed the interventions and outcomes implemented after the RRT screening. Reasons for screening by RRT were sepsis, which accounted for fewer cases, and septic shock, respiratory distress, and cardiogenic shock, which accounted for more cases, in the ICU group. RRT interventions were more frequent in the ICU group, except for extracorporeal membrane oxygenation, renal replacement therapy, application of high-flow nasal cannula, and ultrasonography. Hospital length of stay (LOS) was longer (33 [17-70] vs. 25 [1347], $p=0.011)$, and 28 day-mortality $(22.6 \%$ vs. $14.6 \%$, $p=0.001)$ and in-hospital mortality $(29.8 \%$ vs. $20.1 \%$, $p<0.001)$ were higher in the ICU group.

\section{Factors associated with transfer to ICU}

Multivariate analysis revealed factors associated with ICU transfer (Table 3). After adjusting for confounders, the independent predictors of ICU transfer included SOFA score (OR, 1.281; 95\% CI, 1.184-1.386; $p<0.001$ ), NEWS (OR, 1.065; 95\% CI, 1.006-1.128; $p=0.032$ ), platelet count (OR, 1.002; 95\% CI, 1.001-1.004; $p=0.002)$, and lactate level (OR, 1.161; 95\% CI, 1.074$1.255 ; p<0.001)$.

Factors associated with patients' in-hospital mortality.

Multivariate analysis revealed factors associated with in-hospital mortality (Table 4). The independent predictors of in-hospital mortality included age (OR, 1.029; 95\% CI, 1.013-1.045; $p<0.009)$, screened due to medical reason (OR, 1.799; 95\% CI, 1.115-2.904; $p=0.016$ ), SOFA (OR, 1.119; 95\% CI, 1.042-1.202; $p=0.002$ ), solid tumor (OR, 1.676; 95\% CI, 1.065-2.638; $p=0.026$ ), hematologic malignancy (OR, 3.166; 95\% CI, 1.483$6.760 ; p=0.003$ ), total bilirubin (T-bilirubin; OR, 1.055; 95\% CI, 1.006-1.106; $p=0.027)$, lactate level (OR, 1.166; 95\% CI, 1.078-1.261; $p<0.001$ ), and CRP level (OR, 1.027; 95\% CI, 1.008-1.046; $p=0.005$ ) after adjusting for confounders.

\section{1,218 patients screened on RRT Jan 1, 2016 - Dec 31, 2017}

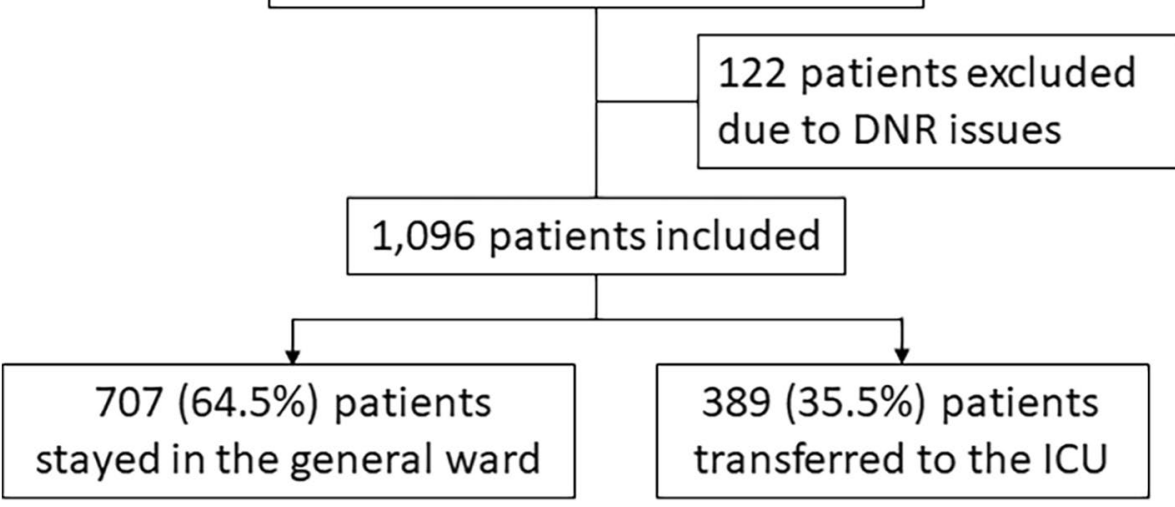

Fig. 1 Flowchart of the patient selection process. RRT, rapid response team; DNR, do not resuscitate; ICU, intensive care unit 
Table 1 Characteristics of the included patients

\begin{tabular}{|c|c|c|c|c|}
\hline & All patients $(n=1096)$ & Ward group $(n=707)$ & ICU group $(n=389)$ & $P$-value \\
\hline Age & $67.4 \pm 14.7$ & $67.8 \pm 14.8$ & $66.5 \pm 14.6$ & 0.145 \\
\hline Male & $675(61.6)$ & $428(60.5)$ & $247(63.5)$ & 0.335 \\
\hline BMI & $22.5 \pm 4.4$ & $22.5 \pm 4.4$ & $22.7 \pm 4.3$ & 0.551 \\
\hline Medical & $792(72.3)$ & $497(70.3)$ & $295(75.8)$ & 0.050 \\
\hline Surgical & $304(27.7)$ & $210(29.7)$ & $94(24.2)$ & 0.050 \\
\hline \multicolumn{5}{|l|}{ Underlying disease } \\
\hline Solid tumor & $182(16.6)$ & $123(17.4)$ & $59(15.2)$ & 0.342 \\
\hline Hematologic malignancy & $54(4.9)$ & $41(5.8)$ & $13(3.3)$ & 0.072 \\
\hline Chronic lung disease & $145(13.2)$ & $109(15.4)$ & $36(9.3)$ & 0.004 \\
\hline Chronic heart disease & $231(21.1)$ & $145(20.5)$ & $86(22.1)$ & 0.535 \\
\hline Chronic hepatobiliary disease & $102(9.3)$ & $55(7.8)$ & $47(12.1)$ & 0.019 \\
\hline Cerebrovascular disease & $182(16.6)$ & $120(17.0)$ & $62(15.9)$ & 0.660 \\
\hline Chronic kidney disease & $116(10.6)$ & $68(9.6)$ & $48(12.3)$ & 0.161 \\
\hline Diabetes & $339(30.9)$ & $213(30.1)$ & $126(32.4)$ & 0.438 \\
\hline Transplantation & $3(0.3)$ & $2(0.3)$ & $1(0.3)$ & 0.938 \\
\hline \multicolumn{5}{|l|}{ Most recent vital sign ${ }^{\mathrm{a}}$} \\
\hline $\mathrm{MBP}, \mathrm{mmHg}$ & $86.7(3.3-102.2)$ & $86.7(73.3-100.0)$ & $86.7(71.7-103.3)$ & 0.568 \\
\hline $\mathrm{HR}$, bpm & $103(88-120)$ & $102(88-116)$ & $106(90-124)$ & 0.002 \\
\hline $\mathrm{RR}, / \mathrm{min}$ & $24(20-30)$ & $24(20-29)$ & $24(20-30)$ & 0.325 \\
\hline $\mathrm{BT},{ }^{\circ} \mathrm{C}$ & $37.4(36.8-38.0)$ & $37.4(36.9-38.0)$ & $37.4(36.8-38.0)$ & 0.238 \\
\hline $\mathrm{SpO} 2, \%$ & $95(91-98)$ & $95(92-98)$ & $95(91-98)$ & 0.238 \\
\hline \multicolumn{5}{|l|}{ Laboratory findings } \\
\hline WBC, $\times 10^{3} / \mathrm{uL}$ & $10.38(7.19-14.40)$ & $9.98(7.1-13.8)$ & $10.8(7.5-16.0)$ & 0.100 \\
\hline $\mathrm{Hb}, \mathrm{g} / \mathrm{dL}$ & $10.3(9.0-12.0)$ & $10.3(9.0-12.1)$ & $10.1(8.8-11.8)$ & 0.448 \\
\hline Platelet, $\times 10^{3} / \mathrm{uL}$ & $182(116-261)$ & $192(123-266)$ & $170(101-250)$ & 0.018 \\
\hline T-bilirubin, mg/dL & $0.71(0.48-1.27)$ & $0.70(0.45-1.19)$ & $0.80(0.50-1.40)$ & 0.018 \\
\hline Creatinine, mg/dL & $0.85(0.62-1.47)$ & $0.78(0.59-1.24)$ & $0.97(0.71-1.90)$ & 0.002 \\
\hline Lactate, mEq/L & $1.8(1.1-3.1)$ & $1.6(1.1-2.5)$ & $2.2(1.2-3.8)$ & 0.213 \\
\hline $\mathrm{CRP}, \mathrm{mg} / \mathrm{dL}$ & $8.8(2.9-16.5)$ & $8.5(2.9-15.7)$ & $9.0(2.8-17.6)$ & $<0.001$ \\
\hline SOFA score & $4(2-6)$ & $3(2-5)$ & $5(3-7)$ & $<0.001$ \\
\hline NEWS score & $8(6-10)$ & $7(6-9)$ & $8(6-10)$ & $<0.001$ \\
\hline $\begin{array}{l}\text { Hospitalization period prior to RRT } \\
\text { activation (Days) }\end{array}$ & $5(1-15)$ & $4(1-14)$ & $6(1-17)$ & 0.268 \\
\hline
\end{tabular}

Data are presented as mean (SD), $\mathrm{n}(\%)$, or median (interquartile range), unless otherwise indicated

${ }^{a}$ It was a vital sign at the time of screening for RRT in the patient and when RRT was activated

$I C U$ Intensive care unit, $B M I$ Body mass index, $M B P$ Mean blood pressure, $H R$ Heart rate, $R R$ Respiratory rate, $B T$ Body temperature, $S p O_{2}$ Saturation by pulse oximetry, SD Standard deviation, WBC White blood cell, $\mathrm{Hb}$ Hemoglobin, CRP C-reactive protein, SOFA Sequential Organ Failure Assessment, NEWS National Early Warning Score, $R R T$ Rapid response team

\section{Discussion}

In this study, $35.5 \%$ of the RRT-screened patients were transferred to the ICU. Patients admitted for medical reasons with an underlying chronic hepatobiliary disease or a higher SOFA score or NEWS were more likely to be admitted to the ICU when screened by RRT. Patients admitted to the ICU had a longer hospital LOS and higher 28-day mortality and in-hospital mortality rates. Higher SOFA, NEWS, platelet count, and lactate level were associated with ICU transfer. ICU transfer was not associated with in-hospital mortality.
RRT has been implemented in several hospitals to facilitate early recognition and treatment of deteriorating patients in wards [3, 15]. Most RRT activation leads to one or more interventions in patients, including additional diagnostic testing, obtaining a venous or central access line, applying oxygen, intubation, use of vasopressors, or supporting cardiopulmonary resuscitation [3, $16,17]$. Interventions were often performed in patients admitted to the ICU in this study because they were more likely to have screened for RRT due to septic shock, respiratory distress, and cardiogenic shock. It is well known 
Table 2 Reasons for screening by RRT, intervention, and outcomes

\begin{tabular}{|c|c|c|c|c|}
\hline & All patients $(n=1096)$ & Ward group $(n=707)$ & ICU group $(n=389)$ & $P$-value \\
\hline \multicolumn{5}{|l|}{ Reason for screening by RRT } \\
\hline Sepsis & $834(76.1)$ & $606(85.7)$ & $228(58.6)$ & $<0.001$ \\
\hline Septic shock & $126(11.5)$ & $48(6.8)$ & $78(20.1)$ & $<0.001$ \\
\hline Respiratory distress & $85(7.8)$ & $39(5.5)$ & $46(11.8)$ & $<0.001$ \\
\hline Cardiogenic shock & $51(4.7)$ & $14(2.0)$ & $37(9.5)$ & $<0.001$ \\
\hline \multicolumn{5}{|l|}{ RRT intervention } \\
\hline ACLS & $41(3.7)$ & $9(1.3)$ & $32(8.2)$ & $<0.001$ \\
\hline ECMO & $3(0.3)$ & $1(0.1)$ & $2(0.5)$ & 0.258 \\
\hline Renal replacement therapy & $17(1.6)$ & $10(1.4)$ & $7(1.8)$ & 0.622 \\
\hline Intubation & $142(13.0)$ & $13(1.8)$ & $129(33.2)$ & $<0.001$ \\
\hline Ventilator & $49(4.5)$ & $6(0.8)$ & $43(11.1)$ & $<0.001$ \\
\hline HFNC & $124(11.3)$ & $99(14.0)$ & $25(6.4)$ & $<0.001$ \\
\hline A-line insertion & $14(1.3)$ & $3(0.4)$ & $11(2.8)$ & 0.001 \\
\hline C-line insertion & $35(3.2)$ & $6(0.8)$ & $29(7.5)$ & $<0.001$ \\
\hline USG & $100(9.1)$ & $64(9.1)$ & $36(9.3)$ & 0.911 \\
\hline Vasopressors & $83(7.6)$ & $22(3.1)$ & $61(15.7)$ & $<0.001$ \\
\hline \multicolumn{5}{|l|}{ Outcomes } \\
\hline Hospital LOS & $28(14-56)$ & $25(13-47)$ & $33(17-70)$ & 0.011 \\
\hline 28 day mortality & $191(17.4)$ & $103(14.6)$ & 88 (22.6) & 0.001 \\
\hline In-hospital mortality & $258(23.5)$ & $142(20.1)$ & $116(29.8)$ & $<0.001$ \\
\hline
\end{tabular}

Data are presented as median (interquartile range) or number (\%), unless otherwise indicated

RRT Rapid response team, ICU Intensive care unit, ACLS Advanced cardiovascular life support, ECMO Extracorporeal membrane oxygenation, HFNC High-flow nasal cannula, A-line Arterial line, C-line Central line, USG Ultrasonography, LOS Length of stay

that these diseases [18-21] require intensive care because of their high severity and require more intervention.

According to a recent review, RRT interventions have improved patient safety $[2,22]$. RRT performance is generally measured in terms of cardiac arrest, unexpected ICU hospitalization, and mortality [23]. Patients with RRT activation tended to have more ICU admissions and a relatively high mortality rate [24]. In this study, the in-hospital mortality rate of RRT-screened patients was $23.5 \%$. The mortality rate has been variously confirmed, ranging from $10.6 \%$ to $42.2 \%[2,5,25,26]$. Several studies have shown that RRT interventions reduce mortality in hospitals $[2,3,22,27]$. Maharaj et al. showed that RRT implementation was associated with an overall hospital mortality reduction in adult patients (relative risk [RR] 0.87, 95\% CI 0.81-0.95, $p<0.001)$ and was associated with a reduction in cardiopulmonary arrest in adults (RR 0.65, 95\% CI 0.61-0.70, $p<0.001$ ) [2]. Chan et. al. showed that RRT activation in adults was associated with a $33.8 \%$ reduction in cardiopulmonary arrest rates outside the ICU (RR 0.66, 95\% CI, 0.54-0.80) but was not associated with lower hospital mortality rates (RR, 0.96; 95\% CI, 0.84-1.09) [27]. However, some studies have shown that RRT interventions do not affect mortality [16]. In the medical early response intervention and therapy study [28], the medical emergency team system did not substantially affect the incidence of cardiac arrest, unplanned ICU admissions, or unexpected death. Therefore, while these results remain controversial, the potential for RRTs to improve meaningful outcomes exists. Therefore, understanding the patient group and the prognosis of patients admitted to the ICU can help improve the effectiveness of RRT.

In this study, higher SOFA score, NEWS, lactate level, and platelet count were factors associated with ICU admission. Higher SOFA score [29-31] and NEWS [14, 32] are well-known factors related to patient severity. Higher scores indicate severe disease in patients; thus, it may have been associated with the patient's ICU admission. Lactate level is known to be related to the severity of systemic hypoperfusion, and high lactate levels are associated with disease severity [33].

Age; screening for medical reasons; higher SOFA score; solid tumor; hematologic malignancy; and higher T-bilirubin, lactate, and CRP levels were associated with in-hospital mortality in this study. Among these factors, higher SOFA scores and lactate levels were also associated with ICU admission. These results were similar to those of other studies. In terms of acute deterioration after more than 7 days of hospitalization, septic shock 
Table 3 Multivariate logistic regression analysis of factors associated with transfer to the ICU

\begin{tabular}{|c|c|c|c|c|c|c|}
\hline & Univa & alysis & & Multiv & Inalysis & \\
\hline & OR & $95 \% \mathrm{Cl}$ & $P$-value & OR & $95 \% \mathrm{Cl}$ & $P$-value \\
\hline Age & 0.994 & $0.986-1.002$ & 0.146 & & & \\
\hline Male & 1.134 & $0.878-1.464$ & 0.335 & & & \\
\hline BMI & 1.009 & $0.979-1.040$ & 0.551 & & & \\
\hline Medical & 1.326 & $1.000-1.759$ & 0.050 & 1.045 & $0.731-1.493$ & 0.811 \\
\hline SOFA score & 1.297 & $1.226-1.371$ & $<0.001$ & 1.281 & $1.184-1.386$ & $<0.001$ \\
\hline NEWS & 1.117 & $1.068-1.167$ & $<0.001$ & 1.065 & $1.006-1.128$ & 0.032 \\
\hline Vital sign & & & & & & \\
\hline MBP & 1.002 & $0.996-1.008$ & 0.547 & & & \\
\hline$H R$ & 1.009 & $1.003-1.014$ & 0.001 & 1.005 & $0.997-1.012$ & 0.210 \\
\hline RR & 1.010 & $0.991-1.029$ & 0.301 & & & \\
\hline Underlying disease & & & & & & \\
\hline Solid tumor & 0.849 & $0.605-1.191$ & 0.343 & & & \\
\hline Hematologic malignancy & 0.562 & $0.297-1.061$ & 0.076 & & & \\
\hline Chronic lung disease & 0.560 & $0.375-0.834$ & 0.004 & 0.742 & $0.458-1.202$ & 0.225 \\
\hline Chronic heart disease & 1.100 & $0.814-1.487$ & 0.535 & & & \\
\hline Chronic hepatobiliary disease & 1.629 & $1.080-2.457$ & 0.020 & 1.189 & $0.708-1.998$ & 0.512 \\
\hline Cerebrovascular disease & 0.927 & $0.663-1.297$ & 0.660 & & & \\
\hline Chronic kidney disease & 1.323 & $0.894-1.958$ & 0.162 & & & \\
\hline Diabetes & 1.111 & $0.851-1.450$ & 0.438 & & & \\
\hline Laboratory findings & & & & & & \\
\hline WBC & 1.010 & $0.997-1.022$ & 0.128 & & & \\
\hline $\mathrm{Hb}$ & 0.977 & $0.923-1.034$ & 0.426 & & & \\
\hline Platelet & 0.999 & $0.998-1.000$ & 0.018 & 1.002 & $1.001-1.004$ & 0.002 \\
\hline T-bilirubin & 1.047 & $1.005-1.090$ & 0.026 & 0.975 & $0.936-1.016$ & 0.224 \\
\hline Creatinine & 1.115 & $1.037-1.198$ & 0.003 & 0.918 & $0.828-1.018$ & 0.104 \\
\hline Lactate & 1.251 & $1.164-1.345$ & $<0.001$ & 1.161 & $1.074-1.255$ & $<0.001$ \\
\hline CRP & 1.009 & $0.995-1.022$ & 0.213 & & & \\
\hline
\end{tabular}

ICU Intensive care unit, OR Odds ratio, CI Confidence interval, BMI Body mass index, SOFA Sequential Organ Failure Assessment, NEWS National Early Warning Score, $M B P$ Mean blood pressure, $H R$ Heart rate, $R R$ Respiratory rate, WBC White blood cell, $H b$ Hemoglobin, $C R P C$-reactive protein

was an independent risk factor for in-hospital mortality and ICU transfer [1,34]. Sepsis and septic shock account for a large proportion of ICU admissions and have been shown to be associated with high in-hospital mortality [35-38]. In a study by Shappell et al., among the deceased patients screened by RRT, more patients were older (median age 72 vs. 66 years), were admitted for noncardiac medical illness (70\% vs. $58 \%$ ), and had a greater median LOS before RRT screening (81 vs. $47 \mathrm{~h}$ ) [39]. In a study by Lee et al., the presence of malignancy was independently associated with in-hospital mortality [40]. Therefore, more careful treatment when patients with these conditions are screened by RRT may help improve patient prognosis.

This study had several limitations. First, this was a retrospective study performed at a single medical center. Second, the possibility of selection bias cannot be ruled out because the data of many patients are recorded in the
EMR by the nurse. However, if the patient had a deteriorating condition, then we trained nurses to check vital signs several times and to input the worst value into the EMR; hence, it was thought that the selection bias might be small. Third, because the RRT was not screened for $24 \mathrm{~h}$, the patient groups that existed at the time when the RRT was not screened were not included in the study. In Fernando's study, patients with acute worsening in the ward assessed by RRT at night (17:00-07:59) had a higher risk of in-hospital death. However, daytime RRT activation is associated with an increased probability of ICU admission [41]. Therefore, exclusion of patients who deteriorated between 11 PM and 7 AM may have affected in-hospital mortality and ICU admission. However, in this study, RRT was not activated only during a shorter time period (11 PM to $7 \mathrm{AM}$ ). As for ICU admission, the impact of time is likely to be small because the intensivists participating in the RRT were also involved in ICU 
Table 4 Multivariate logistic regression analysis of factors associated with in-hospital mortality

\begin{tabular}{|c|c|c|c|c|c|c|}
\hline & \multicolumn{3}{|c|}{ Univariate analysis } & \multicolumn{3}{|c|}{ Multivariate analysis } \\
\hline & OR & $95 \% \mathrm{Cl}$ & $P$-value & OR & $95 \% \mathrm{Cl}$ & $P$-value \\
\hline Age & 1.020 & $1.010-1.031$ & $<0.001$ & 1.029 & $1.013-1.045$ & $<0.001$ \\
\hline Male & 1.246 & $0.931-1.668$ & 0.140 & & & \\
\hline BMI & 1.015 & $0.982-1.050$ & 0.376 & & & \\
\hline Medical & 2.262 & $1.580-3.237$ & $<0.001$ & 1.799 & $1.115-2.904$ & 0.016 \\
\hline SOFA score & 1.235 & $1.169-1.305$ & $<0.001$ & 1.119 & $1.042-1.202$ & 0.002 \\
\hline NEWS & 1.139 & $1.083-1.197$ & $<0.001$ & 1.064 & $0.996-1.138$ & 0.067 \\
\hline \multicolumn{7}{|l|}{ Underlying disease } \\
\hline Solid tumor & 2.075 & $1.474-2.923$ & $<0.001$ & 1.676 & $1.065-2.638$ & 0.026 \\
\hline Hematologic malignancy & 2.993 & $1.719-5.211$ & $<0.001$ & 3.166 & $1.483-6.760$ & 0.003 \\
\hline Chronic lung disease & 1.333 & $0.901-1.973$ & 0.150 & & & \\
\hline Chronic heart disease & 1.252 & $0.899-1.745$ & 0.184 & & & \\
\hline Chronic hepatobiliary disease & 1.475 & $0.943-2.307$ & 0.088 & & & \\
\hline Cerebrovascular disease & 0.934 & $0.639-1.365$ & 0.724 & & & \\
\hline Chronic kidney disease & 1.271 & $0.824-1.961$ & 0.278 & & & \\
\hline Diabetes & 0.981 & $0.725-1.328$ & 0.902 & & & \\
\hline \multicolumn{7}{|l|}{ Laboratory findings } \\
\hline WBC & 1.004 & $0.993-1.016$ & 0.477 & & & \\
\hline $\mathrm{Hb}$ & 0.911 & $0.853-0.973$ & 0.006 & 1.001 & $0.919-1.091$ & 0.974 \\
\hline Platelet & 0.997 & $0.995-0.998$ & $<0.001$ & 0.999 & $0.3997-1.001$ & 0.223 \\
\hline T-bilirubin & 1.106 & $1.049-1.166$ & $<0.001$ & 1.055 & $1.006-1.106$ & 0.027 \\
\hline Creatinine & 1.040 & $0.963-1.123$ & 0.314 & & & \\
\hline Lactate & 1.252 & $1.173-1.338$ & $<0.001$ & 1.166 & $1.078-1.261$ & $<0.001$ \\
\hline CRP & 1.029 & $1.014-1.044$ & $<0.001$ & 1.027 & $1.008-1.046$ & 0.005 \\
\hline Transfer to the ICU & 1.691 & $1.272-2.247$ & $<0.001$ & 1.042 & $0.704-1.542$ & 0.836 \\
\hline
\end{tabular}

OR Odds ratio, CI Confidence interval, BMI Body mass index, SOFA Sequential Organ Failure Assessment, NEWS National Early Warning Score, WBC White blood cell, Hb Hemoglobin, T-bilirubin Total bilirubin, CRP C-reactive protein, ICU Intensive care unit

admission even when the RRT was not activated. Fourth, we conducted a study on a group of patients screened for RRT; therefore, we could not obtain data on patients who were not screened for RRT in the ward and who were transferred to the ICU.

\section{Conclusions}

In this study, $35.5 \%$ of patients screened by RRT were admitted to the ICU. Factors associated with ICU admission were higher SOFA score, NEWS, platelet count, and lactate level. Therefore, close monitoring and transfer to the ICU should be considered when these patients are screened using RRT.

\section{Abbreviations}

RRT: Rapid response team; ICU: Intensive care unit; DNR: Do not resuscitate EMR: Electronic medical record; NEWS: National Early Warning Score; SOFA: Sequential Organ Failure Assessment; IRB: Institutional review board; OR: Odds ratio; Cl: Confidence interval; CRP: C-reactive protein; LOS: Length of stay; RR: Relative risk.

\section{Acknowledgements}

Not applicable.

\section{Authors' contributions}

Conceptualization: S.I.L., J.E.L.; Data curation: S.I.L.; Formal analysis: S.I.L., J.E.L.; Investigation: S.I.L., J.E.L.; Methodology: J.E.L.; Supervision: J.E.L.; Validation: S.I.L., J.E.L.; Visualization: J.E.L.; Writing —original draft: S.IL., J.S.K., Y.J.K., D.H.K, J.E.L.; Writing —review and editing: S.I.L. and J.E.L. All authors read and approved the final version of manuscript.

Funding

The research did not receive any specific grant from funding agencies in the public, commercial, or not-for-profit sectors.

Availability of data and materials

The datasets used and/or analyzed during the current study are available from the corresponding author upon reasonable request.

\section{Declarations}

\section{Ethics approval and consent to participate}

This study was approved by the Institutional Review Board (IRB) of Chungnam National University Hospital (IRB No: CNUH 2019-06-030), and the requirement for informed consent was waived because of the retrospective nature of the study.

\section{Consent for publication}

Not applicable.

\section{Competing interests}

The authors declare that they have no competing interests. 
Received: 17 June 2021 Accepted: 22 January 2022

Published online: 03 February 2022

\section{References}

1. Lee J, Shin Y, Choi E, Choi S, Son J, Jung YK, et al. Impact of hospitalization duration before medical emergency team activation: A retrospective cohort study. PLoS One. 2021;16:e0247066.

2. Maharaj R, Raffaele I, Wendon J. Rapid response systems: a systematic review and meta-analysis. Crit Care. 2015:19:254.

3. Jones DA, DeVita MA, Bellomo R. Rapid-response teams. N Engl J Med. 2011:365:139-46.

4. Neale G, Woloshynowych M, Vincent C. Exploring the causes of adverse events in NHS hospital practice. J R Soc Med. 2001;94:322-30.

5. Lee BY, Hong SB. Rapid response systems in Korea. Acute Crit Care. 2019;34:108-16.

6. Karpman C, Keegan MT, Jensen JB, Bauer PR, Brown DR, Afessa B. The impact of rapid response team on outcome of patients transferred from the ward to the ICU: a single-center study. Crit Care Med. 2013;41:2284-91.

7. Jung B, Daurat A, De Jong A, Chanques G, Mahul M, Monnin M, et al. Rapid response team and hospital mortality in hospitalized patients. Intensive Care Med. 2016;42:494-504.

8. Chang YC, Fang YT, Chen HC, Lin CY, Chang YP, Chen YM, et al. Effect of do-not-resuscitate orders on patients with sepsis in the medical intensive care unit: a retrospective, observational and propensity score-matched study in a tertiary referral hospital in Taiwan. BMJ Open. 2019;9:e029041.

9. Huang CT, Chuang YC, Tsai YJ, Ko WJ, Yu CJ. High Mortality in Severe Sepsis and Septic Shock Patients with Do-Not-Resuscitate Orders in East Asia. PLoS One. 2016;1 1:e0159501.

10 Powell ES, Sauser K, Cheema N, Pirotte MJ, Quattromani E، Avula U, et al. Severe sepsis in do-not-resuscitate patients: intervention and mortality rates. J Emerg Med. 2013:44:742-9.

11. Physicians. RCo. National Early Warning Score (NEWS) 2. [accessed 11-26, 2021]. Available at: https://www.rcplondon.ac.uk/projects/outputs/natio nal-early-warning-score-news-2.

12. Fernando SM, Fox-Robichaud AE, Rochwerg B, Cardinal P, Seely AJE, Perry $\mathrm{JJ}$, et al. Prognostic accuracy of the Hamilton Early Warning Score (HEWS) and the National Early Warning Score 2 (NEWS2) among hospitalized patients assessed by a rapid response team. Crit Care. 2019;23:60.

13 Vincent JL, Moreno R, Takala J, Willatts S, De Mendonca A, Bruining $\mathrm{H}$, et al. The SOFA (Sepsis-related Organ Failure Assessment) score to describe organ dysfunction/failure. On behalf of the Working Group on Sepsis-Related Problems of the European Society of Intensive Care Medicine. Intensive Care Med. 1996;22:707-10.

14. Smith GB, Prytherch DR, Meredith P, Schmidt PE, Featherstone PI. The ability of the National Early Warning Score (NEWS) to discriminate patients at risk of early cardiac arrest, unanticipated intensive care unit admission, and death. Resuscitation. 2013;84:465-70.

15. Aneman A, Frost SA, Parr MJ, Hillman KM. Characteristics and outcomes of patients admitted to ICU following activation of the medical emergency team: impact of introducing a two-tier response system. Crit Care Med. 2015;43:765-73.

16 Chan PS, Khalid A, Longmore LS, Berg RA, Kosiborod M, Spertus JA. Hospital-wide code rates and mortality before and after implementation of a rapid response team. Jama. 2008:300:2506-13.

17. Coventry C, Flabouris A, Sundararajan K, Cramey T. Rapid response team calls to patients with a pre-existing not for resuscitation order. Resuscitation. 2013;84:1035-9.

18 Group SCCT. Incidence of severe sepsis and septic shock in German intensive care units: the prospective, multicentre INSEP study. Intensive Care Med. 2016;42:1980-9.

19 Shankar-Hari M, Harrison DA, Rubenfeld GD, Rowan K. Epidemiology of sepsis and septic shock in critical care units: comparison between sepsis-2 and sepsis-3 populations using a national critical care database. Br J Anaesth. 2017;119:626-36.

20. Huang X, Zhang R, Fan G, Wu D, Lu H, Wang D, et al. Incidence and outcomes of acute respiratory distress syndrome in intensive care units of mainland China: a multicentre prospective longitudinal study. Crit Care. 2020;24:515

21 Jentzer JC. Understanding Cardiogenic Shock Severity and Mortality Risk Assessment. Circ Heart Fail. 2020:13:e007568.
22 Winters BD, Weaver SJ, Pfoh ER, Yang T, Pham JC, Dy SM. Rapid-response systems as a patient safety strategy: a systematic review. Ann Intern Med. 2013:158:417-25

23 Howell MD, Ngo L, Folcarelli P, Yang J, Mottley L, Marcantonio ER, et al. Sustained effectiveness of a primary-team-based rapid response system. Crit Care Med. 2012;40:2562-8.

24. Bannard-Smith J, Lighthall GK, Subbe CP, Durham L, Welch J, Bellomo R, et al. Clinical outcomes of patients seen by Rapid Response Teams: A template for benchmarking international teams. Resuscitation. 2016;107:7-12.

25. Bellomo R, Goldsmith D, Uchino S, Buckmaster J, Hart G, Opdam H, et al. Prospective controlled trial of effect of medical emergency team on postoperative morbidity and mortality rates. Crit Care Med. 2004;32:916-21.

26 Bellomo R, Goldsmith D, Uchino S, Buckmaster J, Hart GK, Opdam H, et al A prospective before-and-after trial of a medical emergency team. Med J Aust. 2003;179:283-7.

27 Chan PS, Jain R, Nallmothu BK, Berg RA, Sasson C. Rapid Response Teams: A Systematic Review and Meta-analysis. Arch Intern Med. 2010;170:18-26.

28 Hillman K, Chen J, Cretikos M, Bellomo R, Brown D, Doig G, et al. Introduction of the medical emergency team (MET) system: a cluster-randomised controlled trial. Lancet. 2005:365:2091-7.

29. Innocenti F, Caldi F, Tassinari I, Meo F, Gandini A, Noto P, et al. SOFA Score prognostic performance among patients admitted to High-Dependency Units. Minerva Anestesiol. 2019;85:1080-8.

30 Karakike E, Kyriazopoulou E, Tsangaris I, Routsi C, Vincent JL, GiamarellosBourboulis EJ. The early change of SOFA score as a prognostic marker of 28-day sepsis mortality: analysis through a derivation and a validation cohort. Crit Care. 2019:23:387.

31 Vincent JL, de Mendonça A, Cantraine F, Moreno R, Takala J, Suter PM, et al. Use of the SOFA score to assess the incidence of organ dysfunction/ failure in intensive care units: results of a multicenter, prospective study. Working group on "sepsis-related problems" of the European Society of Intensive Care Medicine. Crit Care Med. 1998;26:1793-800.

32. Bilben B, Grandal L, Søvik S. National Early Warning Score (NEWS) as an emergency department predictor of disease severity and 90-day survival in the acutely dyspneic patient - a prospective observational study. Scand J Trauma Resusc Emerg Med. 2016;24:80.

33 Okorie ON, Dellinger P. Lactate: biomarker and potential therapeutic target. Crit Care Clin. 2011:27:299-326.

34 Park J, Lee YJ, Hong SB, Jeon K, Moon JY, Kim JS, et al. The association between hospital length of stay before rapid response system activation and clinical outcomes: a retrospective multicenter cohort study. Respir Res. 2021;22:60.

35 Genga KR, Russell JA. Update of Sepsis in the Intensive Care Unit. J Innate Immun. 2017:9:441-55.

36 Perner A, Gordon AC, De Backer D, Dimopoulos G, Russell JA, Lipman J, et al. Sepsis: frontiers in diagnosis, resuscitation and antibiotic therapy. Intensive Care Med. 2016;42:1958-69.

37 Vincent JL, Jones G, David S, Olariu E, Cadwell KK. Frequency and mortality of septic shock in Europe and North America: a systematic review and meta-analysis. Crit Care. 2019:23:196

38 Xie J, Wang H, Kang Y, Zhou L, Liu Z, Qin B, et al. The Epidemiology of Sepsis in Chinese ICUs: A National Cross-Sectional Survey. Crit Care Med. 2020;48:e209-18.

39. Shappell C, Snyder A, Edelson DP, Churpek MM. Predictors of In-Hospital Mortality After Rapid Response Team Calls in a 274 Hospital Nationwide Sample. Crit Care Med. 2018:46:1041-8.

40 Lee J, Ban WH, Kim SW, Kim EY, Han MR, Kim SC. Utilization of a rapid response team and associated outcomes in patients with malignancy. Acute Crit Care. 2020;35:16-23.

41. Fernando SM, Reardon PM, Bagshaw SM, Scales DC, Murphy K, Shen J, et al. Impact of nighttime Rapid Response Team activation on outcomes of hospitalized patients with acute deterioration. Crit Care. 2018;22:67.

\section{Publisher's Note}

Springer Nature remains neutral with regard to jurisdictional claims in published maps and institutional affiliations. 